\title{
Obese Chinese Primary-School Students and Low Self-Esteem: A Cross-Sectional Study
}

\author{
Zhang Xue-Yan, ${ }^{1,2}$ Li Dong-Mei, ${ }^{1}$ Xu Dan-Dan, ${ }^{2}$ and Zhou Le-Shan ${ }^{2,}$ \\ ${ }^{1}$ Qilu Hospital of Shandong University (Qingdao), Qingdao, China \\ ${ }^{2}$ Xiangya Nursing School, Central South University, Changsha, China \\ "Corresponding author: Zhou Le-Shan, Xiangya Nursing School, Central South University, Changsha, China. Tel: +731-82650290, Fax: +731-82650290, E-mail: \\ leshanzhou@csu.edu.cn
}

Received 2015 August 05; Revised 2016 February 13; Accepted 2016 March 09.

\begin{abstract}
Objectives: The aim of this study was to examine several factors related to low self-esteem among obese Chinese primary-school students.

Methods: A cross-sectional study was conducted between June 2009 and June 2010. A total of 1,410 primary-school students (China grades 4 - 6 ) in Changsha city were divided into normal weight $(n=1,084)$, overweight $(n=211)$, and obese groups $(n=115)$ according to world health organization (WHO) growth standards for body mass index (BMI). The students were assessed using the self-esteem scale(SES) and a general situation questionnaire. Caregivers completed questionnaires about their child's weight status. Self-esteem levels were explored; any factors related to low self-esteem were analyzed using logistic regression analysis.

Results: The average self-esteem score among overweight or obese primary-school students was found to be lower than that of normal-weight students. The proportion of students with low self-esteem in the obese group was more than that in the normalweight and overweight groups. Multiple logistic regression analysis showed that obesity status (odds ratio [OR],3.74; 95\% confidence interval [CI],2.25 - 6.22), overweight status (OR, 2.60; 95\% CI,1.71-3.95), obesity considered by children's grandparents (OR, 1.76; $95 \% \mathrm{CI}$, 1.05 - 2.96), dissatisfaction with height (OR, 1.55; $95 \% \mathrm{CI}, 1.11$ - 2.18), and dissatisfaction with weight (OR, 1.45; 95\% CI, 1.05-2.01) were the risk factors for low self-esteem for primary-school students, while satisfaction with academic performance was a protective factor (OR, 0.22; 95\% CI, 0.07 - 0.71).

Conclusions: For Chinese primary-school students, low self-esteem is associated with higher weight status and self-perceived body shape and academic performance. In addition, grandparental opinion of a child's weight also contributes to low self-esteem.
\end{abstract}

Keywords: Self-Esteem, Primary-School Student, Obesity, Overweight, China

\section{Background}

Self-esteem reflects the personal appraisal of one's own value (1) and is directly associated with children's social, emotional, behavioral, and mental development $(2,3)$. Low self-esteem is a risk factor among children that may contribute to serious outcomes such as suicidal behavior (4), whereas students with higher self-esteem are generally considered to have better physical health (5). It is thus crucial to facilitate positive self-esteem among primary-school students. Children's self-esteem levels are not as stable as they are among adults and are susceptible to the influence of various external factors. Biddle et al. found that physical activity was associated with better self-esteem (6). According to Tin and colleagues, television-viewing hours showed an inverted J-shaped relation to children's self-esteem levels (7). In addition, many researchers have explored the relationships between children's self-esteem and peer teasing, academic performance, and physical appearance.

Childhood obesity is now a major social problem in both developed and developing countries whose prevalence is continuing to increase at a dramatic rate (8, 9). Childhood obesity is associated with many long-term health consequences (10). While it also appears to place children at increased risk for psychological problems, research into the association between childhood obesity and self-esteem has yielded mixed results (11-13). This might be because self-esteem can be measured globally or in relation to one specific domain (14); as such, overweight or obese children in some samples may show decreases in a specific domain of self-esteem $(15,16)$. Several studies have also explored different factors related to obese children's self-esteem status, including gender, age, peer teasing, disturbed eating behaviors, television viewing, and parents' perceptions of their children's body or weight status $(2,3$, 5-7). In addition to self-esteem scores, the percentage of children with different self-esteem levels is another important parameter to consider. Based on the different methods that are used, self-esteem may be divided into high, 
medium, and low levels.

In China, while several studies have found significant differences in self-esteem scores among junior students between normal-weight and obese children, few have attempted to explore the self-esteem status of obese Chinese primary-school students.

\section{Objectives}

This study was undertaken to assess the present selfesteem situation and to investigate related factors. Our findings may contribute to the regulation of weight as a way to optimize self-esteem among primary-school students.

\section{Methods}

\subsection{Subjects}

Between June 2009 and August 2009, five districts of Changsha city were randomly chosen; two primary schools in each district were randomly selected. Three classes from grades 4 - 6 were then evaluated in the selected primary schools. The inclusion criterion was that students were willing to complete the self-esteem status survey. The exclusion criteria included the existence of 1 , chronic diseases or severe mental disorders that caused cognitive and learning problems (such as schizophrenia); 2 , severe heart or physical dysfunctions; and 3, those who were suffering from acute diseases and had taken glucocorticoids in the previous two weeks. According to world health organization (WHO) growth standards for body mass index (BMI), (17) a sex-specific BMI (by age) at or above the 85th percentile is defined as overweight, at or above the $95^{\text {th }}$ percentile is defined as obese, and at or below the fifth percentile is defined as underweight. For example, the normal BMI range of a ten-year-old girl is $13.9-19.1$; a BMI $\geq 19.1$ is defined as overweight, and a BMI $\geq 21.1$ is defined as obese.

The subjects were divided into four groups: obese, overweight, normal weight, and underweight. Parental written informed consent was obtained for all participants. The research was approved by the regional committee for medical research ethics in China as well as the local education and health-administrative department.

The survey was conducted at the same time as the annual student health inspection. Participants were selected strictly. Group researchers and school health teachers were all trained according to a standard protocol. The questionnaires were distributed to the families through the local schools; the children completed the survey at school, while the parents' surveys were returned the next day.

\subsection{Instruments and Procedure}

\subsection{1. $B M I$}

The BMI of each participant was calculated using objective height and weight measurements collected by trained researchers.

\subsubsection{Self-Esteem}

Self-esteem status was assessed using the Chinese version of the rosenberg self-esteem scale (RSES), which was completed by the children (18). The survey contained ten items; the response options for each item were extremely true, true, false, or extremely false (scored $4 / 3 / 2 / 1$, respectively). The total scores were between 10 and 40 ; higher scores indicated higher self-esteem. The psychometric properties of the Chinese version of RSES have been empirically tested; its internal consistency was found to have alpha coefficients of 0.77 (19). There are three different methods for determining self-esteem levels: statistically, psychologically, and mathematically; the first two are the most frequently used methods. Because the psychological method is not suitable for correctly evaluating selfesteem distribution, this research instead adopted the statistical method. Self-esteem scores between mean \pm standard deviation (SD) were defined as medium, scores between mean $+1 S D$ and mean $+3 S D$ were defined as high, and scores between mean -1SD and mean -3SD were defined as low.

\subsubsection{Caregivers' Evaluation of Child's Body Status}

The children's caregivers included the children's parents and grandparents. Caregivers' evaluations of child's body status were assessed by questions such as, Do you think your child is obese? and Do you think he/she is healthy? The only responses were yes or no. Although this evaluation does not reflect the children's actual body status, it can be used to assess if parents' or grandparents' perception affects children's self-esteem.

\subsubsection{Academic Performance}

Ranks were used to assess students' perceived academic performance. Three degrees were used to assess their academic performance in class: high, moderate, and low. Each student's performance was categorized according to his or her average academic test-score ranks. The rank orders (1-3) were then used for analysis in this study.

\subsubsection{General Situation Questionnaire}

This questionnaire (completed by the children) aimed to evaluate sociodemographic data such as gender, family population (i.e., if the child was an only child, or if the child was from a single-parent household), whether the 
parents' relationship with each other was good or not, life and learning conditions, learning stress, academic performance, and self-perceived body shape (height, weight, and obesity). We selected items that may be indicators of low self-esteem. Each item had only two answers: yes or no. For example, if a child answered yes to the question, Have you ever felt you are too fat? that child was scored 1 ; if not, the child was scored 0 . For gender, males were scored 0 and females 1 .

\subsection{Statistical Analysis}

All analyses were conducted using PASW Statistics, version 18.0.0 (SPSS Inc., Chicago, IL, 2009). Data were presented as mean \pm SD and proportions. Independent sample t-tests and analysis of variance (ANOVA) with posthoc Bonferroni tests were used to investigate self-esteem score differences between male and female students and between normal-weight, overweight, and obese students. Correlation analysis was used to explore the relationship between self-esteem scores and children's BMI. Pearson's chi-square tests were employed to calculate whether differences between the percentages of the three weight groups with different self-esteem levels were significant.

We conducted multiple-factor non-conditional logistic regression analyses to identify any factors associated with low self-esteem in children; overweight, obese, and sociodemographic data (child and family general situation, academic performance, self-perceived body shape, parents' and grandparents' perception of child's weight status) were entered as predictors. Low self-esteem was the dependent variable. The selected predictors were entered backward in the model.

All $\mathrm{P}$ values presented were two tailed; $\mathrm{P}<0.05$ was considered to be statistically significant.

\section{Results}

\subsection{Sample Characteristics}

In total, the study included 1,637 primary school students in 30 classes. Because 31 students did not participate, a total of 1,606 students completed the questionnaires. After rejecting 176 unsatisfactory questionnaires due to missing data, the remaining 1,430 students were divided into obese, overweight, normal-weight, and underweight groups. Because there were only 20 underweight students ( 8 males, 12 females), which was an insufficient number to do the subsequent survey, no underweight students were included in this study (nor are they included in any of the tabulations). Thus, the final sample was comprised of 1,410 primary-school students. The age of the sample was between 9 and 12 years; 76.9\% $(n=1,084)$ of the children were of normal weight, $14.9 \%(n=211)$ were overweight, and $8.2 \%(n=115)$ were obese. For self-esteem scores, 28.2\% $(n=398)$ of the children had high self-esteem, 54.5\% $(\mathrm{n}=768)$ had medium self-esteem, and17.3\% $(\mathrm{n}=244)$ suffered from low self-esteem.

Self-esteem scores of male and female students with different BMIs

The average self-esteem score of the entire sample was 22.7 \pm 4.2 . We found statistically significant differences between children with different BMI categories vis-à-vis selfesteem scores $(\mathrm{F}=12.41, \mathrm{P}<0.001)$. The post-hoc test result showed that the self-esteem scores of the overweight and obese groups were lower than those of the normalweight students (21.9 \pm 4.1 and $21.5 \pm 4.5$ vs. $23.0 \pm 4.2$, respectively; $\mathrm{P}<0.01)$. We also observed a negative correlation between self-esteem scores and $\mathrm{BMI}(\mathrm{r}=-0,120, \mathrm{P}<0.05)$. The mean scores of the male and female students for selfesteem and the corresponding significance levels are presented in Table 1 . The results related to differences among genders showed no statistical significance. Obese male children demonstrated lower self-esteem than overweight and normal-weight children, while overweight and obese female children scored lower than their normal-weight peers.

\subsection{Self-Esteem Level in Normal-Weight, Overweight, and Obese Students}

Table 2 presents the percentage of normal-weight, overweight, and obese children and their self-esteem levels. Chi-square tests showed that more obese children suffered from low self-esteem.

\subsection{Factors Associated With Low Self-Esteem in Children}

Table 3 shows the results of the multifactor logistic regression analyses for low self-esteem; the results are only reported for independent variables for which significant relationships were identified. Obesity (OR, 3.741; 95\% CI, 2.25 - 6.22), overweight (OR, 2.60; 95\% CI, 1.71 - 3.95), grandparents' evaluation as obese (OR, 1.76; 95\% CI, 1.05 - 2.96), children's dissatisfaction with their height (OR, 1.55; 95\% CI, 1.11 - 2.18), and dissatisfaction with their weight (OR, 1.45; 95\% CI, 1.05 - 2.01) were risk factors for low self-esteem, while children's satisfaction with their academic performance (OR, 0.22; 95\% CI, 0.07 - 0.71) was identified as a protective factor.

\section{Discussion}

In this sample, overweight and obese children demonstrated significantly lower self-esteem levels than normalweight children, which is in line with previous studies' 
Table 1. Self-Esteem Scores of Male and Female Students With Different BMI ${ }^{\mathrm{a}}$

\begin{tabular}{|c|c|c|c|c|c|c|}
\hline \multirow[t]{2}{*}{ Group } & \multicolumn{2}{|c|}{$\mathbf{N}$} & \multicolumn{2}{|c|}{ Scores } & \multirow[t]{2}{*}{$\mathbf{t}$} & \multirow[t]{2}{*}{$\mathbf{P}$} \\
\hline & Male & Female & Male & Female & & \\
\hline Normal & 557 & 527 & $23.1 \pm 4.0$ & $23.0 \pm 4.4$ & 0.57 & 0.6 \\
\hline Overweight & 121 & 90 & $22.1 \pm 4.3$ & $21.5 \pm 3.9$ & 1.01 & 0.3 \\
\hline Obese & 71 & 44 & $21.4 \pm 5.0$ & $21.5 \pm 3.5$ & -0.12 & 0.9 \\
\hline $\mathbf{P}$ & - & - & 0.001 & 0.003 & - & - \\
\hline
\end{tabular}

${ }^{\mathrm{a}}$ Values are expressed as mean $\pm \mathrm{SD}$

Table 2. Percentages of Normal, Overweight, and Obese Students With High, Medium, and Low Levels of Self-Esteem, $\mathrm{n}(\%)$

\begin{tabular}{|c|c|c|c|c|c|}
\hline Self-Esteem level ${ }^{\mathbf{b}}$ & Normal & Overweight & Obese & $\chi^{2}$ & $\mathbf{P}$ \\
\hline High & $319(29.4)$ & $57(27.0)$ & $22(19.1)$ & 5.622 & 0.06 \\
\hline Medium & $593(54.7)$ & $109(51.7)$ & $66(57.4)$ & 1.092 & 0.6 \\
\hline Low & $172(15.9)$ & $45(20.9)$ & $27(23.5)$ & 7.014 & 0.03 \\
\hline
\end{tabular}

${ }^{\mathrm{a}}$ Values are expressed as No. (\%).

${ }^{\mathrm{b}}$ Self-esteem level was divided statistically by the mean, 1SD, and 3SD of scores.

Table 3. Logistic Regression Analyses of Factors Related to Low Self-Esteem in Children ${ }^{\mathrm{a}, \mathrm{b}}$

\begin{tabular}{|c|c|c|c|c|c|c|c|}
\hline Predictors $^{c}$ & B & S.E. & Wald & df & $\mathbf{P}$ & OR & 95\% CI \\
\hline Obese & 1.319 & 0.260 & 25.811 & 1 & $<0.001$ & 3.741 & $2.249-6.223$ \\
\hline Overweight & 0.954 & 0.214 & 19.801 & 1 & $<0.001$ & 2.596 & $1.705-3.951$ \\
\hline Grandparents' evaluation as obese & 0.567 & 0.265 & 4.584 & 1 & 0.03 & 1.763 & $1.049-2.963$ \\
\hline Dissatisfaction with height & 0.439 & 0.173 & 6.442 & 1 & 0.01 & 1.551 & $1.105-2.177$ \\
\hline Dissatisfaction with weight & 0.373 & 0.167 & 4.965 & 1 & 0.03 & 1.451 & $1.046-2.014$ \\
\hline Satisfaction with academic performance & -1.507 & 0.595 & 6.403 & 1 & 0.01 & 0.222 & $0.069-0.712$ \\
\hline
\end{tabular}

${ }^{a}$ Results are only reported for independent variables for which significant relationships were identified. The dependent variable was low self-esteem; in addition to the predictors shown in this table, the independent variables also included sociodemographic data such as gender, single child or not, parents' relationship, and so on. ${ }^{\mathrm{b}} \alpha_{\text {entry }}=0.05, \alpha_{\text {removal }}=0.10$.

${ }^{\mathrm{c}}$ Responses for predictors are yes and no (yes $=1$, no $=0$ ).

findings (11, 12). Although self-esteem can include global or domain-specific measures, this study focused on global self-esteem. Studies that measure domain-specific selfesteem have found that children who are overweight or obese are more likely to have low self-esteem related to their physical appearance $(15,16)$. Similarly, one group of researchers hypothesized that body dissatisfaction may mediate the association between obesity and self-esteem (20), which indicates that overweight or obese students are more concerned about their weight and shape than normal-weight students. In China, although the idea that overweight or obesity correlates with good health still exists (21-23), in the rapidly Westernizing and highly com- petitive Chinese society, Western ideals about weight and slim body shape are now becoming widely accepted (22). For primary-school students, society focuses on slimness as a body ideal, especially for little girls. They are more aware of their shape and image than boys; therefore, it is not surprising that the overweight female students in our study reported lower self-esteem than the normal-weight students, while the overweight male students did not.

In addition to self-esteem scores, the percentage of primary-school students with different self-esteem levels is another important parameter to consider. There are three different methods for determining self-esteem levels: statistically, psychologically, and mathematically. Statistical 
division is based on the cut-off point of mean and SD. Table 2 shows that for the low-self-esteem group, more obese children suffered low self-esteem than their overweight or normal-weight peers.

Our findings also suggest that obesity was the strongest predictor of low self-esteem: obese students were more than twice as likely to suffer from low selfesteem compared to normal-weight students, which is similar to the findings of a previous study (24). According to one longitudinal study (11). Children who are obese at baseline will have almost twice the likelihood of reporting low self-esteem four years later, which indicates that a strong relationship exists between obesity and low selfesteem. Since childhood obesity impairs body movement and the ability to compete athletically, several studies have found that obese children are more likely to be teased by their peers $(25,26)$. Another study found that obese white adolescents have fewer friends and less reciprocation from a best friend than non-obese adolescents (27). It is likely that these unpleasant experiences may damage their self-esteem more seriously than is the case with normal-weight students, and overweight students will become more concerned about their weight and shape than normal-weight children (28). In our study, overweight children were 2.59 times more likely to suffer from low self-esteem compared to their normal-weight peers.

We found that children's perceptions of their weight and height were associated with low self-esteem. Dissatisfaction with height and weight were found to be risk factors for low self-esteem. Another study (by Huang et al.) involving Chinese students demonstrated that self-reporting as overweight had a clear and measurably adverse impact on mental health (29). As Western culture and traditional Chinese culture collide, being too fat or too thin is no longer socially accepted $(21,22)$. Lam et al. (30) investigated the relationship between sociocultural influences in promoting thinness, age, and BMI to body dissatisfaction and dieting among 294 Hong Kong adolescent girls; the authors found that with the modernizing of traditional cultures, a culture of thinness appeared to be associated with weight-loss efforts among girls in modernizing cultures independently of body dissatisfaction. Our sample consisted of 9 - 12 years old, which is an age range in which secondary sex characteristics begin to emerge. Children of this age become sensitive to their body shape and may have negative attitudes toward their height and weight. Studies suggest that inaccurate perception of one's weight (rather than obesity per se) may mediate the relationship between actual weight and poor psychological state $(31,32)$. This observation reminds us of the importance of guiding students to evaluate themselves objectively.

One of the most interesting findings from the present study was the grandparents' evaluation of obesity as a risk factor for low self-esteem. Previous studies have attached considerable importance to the association between parents' perceptions and children's self-esteem; they have reported that parental evaluation of their children as being overweight or obese is associated with a low appearance of self-esteem $(15,16,33)$. Our study also identified a relationship between grandparents' perceptions of children's weight status and their self-esteem. With the trend of an aging population in China, the three-generation family is becoming more and more common (34). Grandparents often become children's primary caretakers in threegeneration families (35). Because the number of children who are reared by their grandparents has increased in recent years (36) especially among those whose parents are too busy working to care for them. Those children may rely on their grandparents more than their parents. This means that grandparents' perceptions of their weight status may be an additional burden on the children's selfesteem.

Satisfaction with academic performance was the only protective factor against low self-esteem found in our study. Wang et al. also found that school performance positively affected self-esteem (24). Academic success reflects the comprehension and mastery of learning material, as well as consistent school attendance and participation $(36,37)$. Academics are the most important task for students, and successful performance can produce positive feedback from teachers and peers; students' satisfaction with their academic performance can thus improve their self-esteem to some extent. This may be good for students' self-esteem, especially those who are obese, since they can improve their self-image by working hard academically.

One strength of the present study was that we explored both self-esteem levels and related factors. While previous studies found that obese children are likely to suffer from low self-esteem, our study confirmed this finding and identified several factors related to low self-esteem in Chinese culture. Future research should focus on ways to improve the self-esteem of primary-school students who cope with these factors.

This study does have several limitations. The sample included only Chinese primary-school students; our results thus cannot be generalized to junior students or to children of other cultural backgrounds. Because of the small number of underweight students in our sample, regression analyses were performed on normal-weight, overweight, and obese samples; it is possible that different results would have been obtained had underweight students been included in the sample. Because this was a crosssectional study, we were not able to draw any inferences about causality. Although we selected several possible pre- 
dictors for low self-esteem scores, information on certain relevant predictors, such as peer teasing, was not available. Longitudinal research should be carried out to clarify more precise relationships between obesity and selfesteem and between low self-esteem and related factors.

\subsection{Conclusion}

For Chinese primary-school students, our study found that more obese children suffered from low self-esteem than their overweight and normal-weight peers. Higher weight status, self-perceived body shape, academic performance, and grandparental opinion of a child's weight were all influencing factors for low self-esteem. This study highlights the need to pay attention to obese children's psychological problems and to reduce their exposure to risk factors for low self-esteem.

\section{Acknowledgments}

Funding was provided by the China medical board (CMB; no. 10-020-201102) and the hunan ministry of science and technology (no. 2012WK3058). We would also like to thank all of the students who participated in this study.

\section{Footnotes}

Authors' Contribution: Zhou Le-Shan conceptualized the study and was principal investigator; Li Dong-Mei was responsible for data collection; Zhang Xue-Yan and Xu DanDan were responsible for data analysis and for writing the manuscript.

Conflict of Interest: The authors have no conflicts of interest to report.

\section{References}

1. Mann M, Hosman CM, Schaalma HP, de Vries NK. Self-esteem in a broad-spectrum approach for mental health promotion. Health Educ Res. 2004;19(4):357-72. doi: 10.1093/her/cyg041. [PubMed:15199011].

2. Donnellan MB, Trzesniewski KH, Robins RW, Moffitt TE, Caspi A. Low self-esteem is related to aggression, antisocial behavior, and delinquency. Psychol Sci. 2005;16(4):328-35. doi: 10.1111/j.09567976.2005.01535.x. [PubMed: 15828981].

3. Wild LG, Flisher AJ, Bhana A, Lombard C. Associations among adolescent risk behaviours and self-esteem in six domains. J Child Psychol Psychiatry. 2004;45(8):1454-67. doi: 10.1111/j.1469-7610.2004.00851.x. [PubMed: 15482505].

4. McGee R, Williams S, Nada-Raja S. Low self-esteem and hopelessness in childhood and suicidal ideation in early adulthood. J Abnorm Child Psychol. 2001;29(4):281-91. [PubMed: 11523834].

5. Kermode S, MacLean D. A study of the relationship between quality of life, health and self-esteem. Aust J Adv Nurs. 2001;19(2):33-40. [PubMed: 11845707].

6. Biddle SJ, Asare M. Physical activity and mental health in children and adolescents: a review of reviews. Br J Sports Med. 2011;45(11):886-95. doi: 10.1136/bjsports-2011-090185. [PubMed: 21807669]
7. Tin SP, Ho DS, Mak KH, Wan KL, Lam TH. Association between television viewing and self-esteem in children. J Dev Behav Pediatr. 2012;33(6):479-85. doi: 10.1097/DBP.0b013e31825ab67d. [PubMed 22772822].

8. Jackson-Leach R, Lobstein T. Estimated burden of paediatric obesity and co-morbidities in Europe. Part 1. The increase in the prevalence of child obesity in Europe is itself increasing. Int J Pediatr Obes 2006;1(1):26-32. [PubMed: 17902212].

9. El-Bayoumy I, Shady I, Lotfy H. Prevalence of obesity among adolescents (10 to 14 years) in Kuwait. Asia Pac J Public Health. 2009;21(2):1539. doi:10.1177/1010539509331786. [PubMed: 19190003].

10. Abdelalim A, Ajaj N, Al-Tmimy A, Alyousefi M, Al-Rashaidan S, Hammoud MS, et al. Childhood obesity and academic achievement among male students in public primary schools in Kuwait. Med Princ Pract. 2012;21(1):14-9. doi: 10.1159/000331792. [PubMed: 22024698].

11. Wang F, Wild TC, Kipp W, Kuhle S, Veugelers PJ. The influence of childhood obesity on the development of self-esteem. Health Rep. 2009;20(2):21-7. [PubMed: 19728582]

12. Walpole B, Dettmer E, Morrongiello B, McCrindle B, Hamilton J. Motivational interviewing as an intervention to increase adolescent self-efficacy and promote weight loss: methodology and design. BMC Public Health. 2011;11:459. doi: 10.1186/1471-2458-11-459. [PubMed: 21663597].

13. Russell-Mayhew S, McVey G, Bardick A, Ireland A. Mental health, wellness, and childhood overweight/obesity.JObes. 2012;2012:281801. doi: 10.1155/2012/281801. [PubMed: 22778915].

14. Wardle J, Cooke L. The impact of obesity on psychological wellbeing. Best Pract Res Clin Endocrinol Metab. 2005;19(3):421-40. doi: 10.1016/j.beem.2005.04.006. [PubMed: 16150384].

15. Young-Hyman D, Schlundt DG, Herman-Wenderoth L, Bozylinski K. Obesity, appearance, and psychosocial adaptation in young African American children. J Pediatr Psychol. 2003;28(7):463-72. [PubMed: 12968038]

16. Danielsen YS, Stormark KM, Nordhus IH, Maehle M, Sand L, Ekornas $\mathrm{B}$, et al. Factors associated with low self-esteem in children with overweight. Obes Facts. 2012;5(5):722-33. doi:10.1159/000338333. [PubMed: 23108439].

17. Who . WHO child growth standards: methods and development World Health Organization; 2007.

18. Ho KY, Li WH, Chan SS. The effect of poverty and income disparity on the psychological well-being of Hong Kong children. Public Health Nurs. 2015;32(3):212-21. doi: 10.1111/phn.12147. [PubMed: 25088868]

19. Shek DT. Paternal and maternal influences on the psychological wellbeing, substance abuse, and delinquency of Chinese adolescents experiencing economic disadvantage. J Clin Psychol. 2005;61(3):219-34. doi: 10.1002/jclp.20057. [PubMed: 15515041].

20. Shin NY, Shin MS. Body dissatisfaction, self-esteem, and depression in obese Korean children. J Pediatr. 2008;152(4):502-6. doi 10.1016/j.jpeds.2007.09.020. [PubMed:18346504].

21. Lai KY. Anorexia nervosa in Chinese adolescents-does culture make a difference?.J Adolesc. 2000;23(5):561-8. doi: 10.1006/jado.2000.0343. [PubMed: 11073697].

22. Lee AM, Lee S. Disordered eating and its psychosocial correlates among Chinese adolescent females in Hong Kong. Int J Eat Disord. 1996;20(2):177-83. doi: 10.1002/(SICI)1098-108X(199609)20:2<177::AIDEAT8>3.0.CO;2-D. [PubMed: 8863070].

23. Lee $S$, Lee AM. Disordered eating in three communities of China: a comparative study of female high school students in hong kong, Shenzhen, and rural hunan. Int J Eat Disord. 2000;27(3):317-27. [PubMed: 10694718].

24. Wang F, Veugelers PJ. Self-esteem and cognitive development in the era of the childhood obesity epidemic. Obes Rev. 2008;9(6):615-23. doi: 10.1111/j.1467-789X.2008.00507.x. [PubMed: 18647242]. 
25. Fox CL, Farrow CV. Global and physical self-esteem and body dissatisfaction as mediators of the relationship between weight status and being a victim of bullying. J Adolesc. 2009;32(5):1287-301. doi: 10.1016/j.adolescence.2008.12.006. [PubMed: 19157531].

26. Li W, Rukavina P. The nature, occurring contexts, and psychological implications of weight-related teasing in urban physical education programs. Res Q Exerc Sport. 2012;83(2):308-17. doi: 10.1080/02701367.2012.10599862. [PubMed: 22808717].

27. Cunningham SA, Vaquera E, Long JL. Race, ethnicity, and the relevance of obesity for social integration. Ethn Dis. 2012;22(3):317-23. [PubMed: 22870575].

28. Allen KL, Byrne SM, Blair EM, Davis EA. Why do some overweight children experience psychological problems? The role of weight and shape concern. Int J Pediatr Obes. 2006;1(4):239-47. [PubMed: 17907331].

29. Huang L, Tao FB, Wan YH, Xing C, Hao J, Su PY, et al. Self-reported weight status rather than BMI may be closely related to psychopathological symptoms among Mainland Chinese adolescents. J Trop Pediatr. 2011;57(4):307-11. doi: 10.1093/tropej/fmp097. [PubMed:19797398].

30. Lam TH, Lee SW, Fung S, Ho SY, Lee PW, Stewart SM. Sociocultural influences on body dissatisfaction and dieting in Hong Kong girls. Eur Eat Disord Rev. 2009;17(2):152-60. doi: 10.1002/erv.900. [PubMed
18792894].

31. Franklin J, Denyer G, Steinbeck KS, Caterson ID, Hill AJ. Obesity and risk of low self-esteem: a statewide survey of Australian children. Pediatrics. 2006;118(6):2481-7. doi: 10.1542/peds.2006-0511. [PubMed: 17142534].

32. Yamamoto C, Uemoto M, Shinfuku N, Maeda K. The usefulness of body image tests in the prevention of eating disorders. Kobe J Med Sci. 2007;53(3):79-91. [PubMed: 17684439].

33. Davison K, Birch LL. Weight status, parent reaction, and self-concept in five-year-old girls. Pediatrics. 2001;107(1):46-53.

34. Zhigang G. Study on changes of china family households in recent years. Chinese J Population Sci. 2008;3:2-10.

35. Jingxiong J. Intervention for childhood obesity in Beijing, China. 173. ;. p. 49.

36. Lu Y, Zhu P, Gao R, Liang ZZ, Huang W, Tao FB. Association of rearing patterns with diet and temperament traits among infants in urban areas. Chinese J contem pediat. 2012;14(1):48-53.

37. Hussaini AE, Nicholson LM, Shera D, Stettler N, Kinsman S. Adolescent obesity as a risk factor for high-level nicotine addiction in young women. J Adolesc Health. 2011;49(5):511-7. doi: 10.1016/j.jadohealth.2011.04.001. [PubMed: 22018566]. 